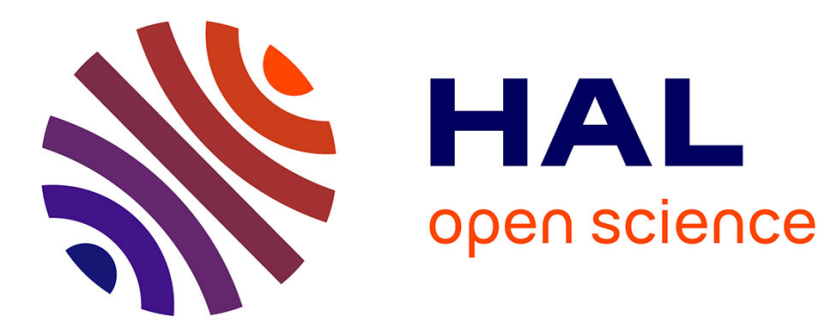

\title{
Synthesis of 3,6-Divinyl-1,2,4,5-Tetrazine, the First Member of the Elusive Vinyltetrazine Family
}

Stéphanie Pican, Vincent Lapinte, Jean-François Pilard, Eric Pasquinet, Lionel Beller, Laurent Fontaine, Didier Poullain

\section{- To cite this version:}

Stéphanie Pican, Vincent Lapinte, Jean-François Pilard, Eric Pasquinet, Lionel Beller, et al.. Synthesis of 3,6-Divinyl-1,2,4,5-Tetrazine, the First Member of the Elusive Vinyltetrazine Family. SYNLETT, 2009, 5, pp.731-734. 10.1055/s-0028-1087818 . hal-00379187

\section{HAL Id: hal-00379187 https://hal.science/hal-00379187}

Submitted on 27 Apr 2009

HAL is a multi-disciplinary open access archive for the deposit and dissemination of scientific research documents, whether they are published or not. The documents may come from teaching and research institutions in France or abroad, or from public or private research centers.
L'archive ouverte pluridisciplinaire HAL, est destinée au dépôt et à la diffusion de documents scientifiques de niveau recherche, publiés ou non, émanant des établissements d'enseignement et de recherche français ou étrangers, des laboratoires publics ou privés. 


\title{
Print out this copy of the sample article.
}

\section{Synthesis of 3,6-Divinyl-1,2,4,5-Tetrazine, the First Member of the Elusive Vinyltetrazine Family}

\author{
Stéphanie Pican, ${ }^{\mathrm{a}}$ Vincent Lapinte, ${ }^{\mathrm{a}, \mathrm{c}}$ Jean-François Pilard, ${ }^{\mathrm{a} *}$ Eric Pasquinet, ${ }^{\mathrm{b}}$ Lionel Beller, ${ }^{\mathrm{b}}$ Laurent Fontaine, ${ }^{\mathrm{a}}$ Didier \\ Poullain ${ }^{\mathrm{b}}$ \\ ${ }^{a}$ UCO2M - Chimie des polymères, UMR6011 CNRS, Université du Maine, \\ Avenue O. Messiaen, 72085 Le Mans Cedex 09, France
}

${ }^{\mathrm{b}}$ CEA, DAM, LE RIPAULT, F-37260 Monts, France

' Institut Charles Gerhardt, UMR5253 CNRS, IAM, Université Montpellier II, Place E. Bataillon, 34095 Montpellier Cedex 5, France

Fax: +33247345142 .

E-mail: jean-francois.pilard@univ-lemans.fr.

Received: The date will be inserted once the manuscript is accepted.

\begin{abstract}
The synthesis of the first vinyltetrazine derivative is described. 3,6-Divinyl-1,2,4,5-tetrazine was obtained following a methodology involving cyclization from an imidate and use of 2phenylsulfonylethyl groups as masked vinyl entities. The first properties of this unique compound are reported.
\end{abstract}

Key words: Azo compounds; Condensation; Cyclizations; Fusedring systems; Heterocycles.

Polymers based on nitrogen-rich heterocycles have been the subject of numerous studies, owing to their specific characteristics, such as thermal stability and energetic properties. Hence, a substantial amount of work has been devoted to the synthesis of vinylsubstituted azaheterocycles. For example, 2- and 5vinyltetrazole derivatives have been obtained and polymerized to yield polyvinyltetrazoles as energetic binding agents for jet engine fuels, gunpowders or explosives. In the triazoles series, both 4(5)-vinyl-1,2,3triazoles and 1- or 4-vinyl-1,2,4-triazoles are known and the resulting polymers were described. ${ }^{2}$ The same also applies to vinyl derivatives of 1,3,5-triazines and polymers thereof. ${ }^{3}$ Among all this work on high-nitrogen polymers, it appeared that tetrazine-containing polymers had been largely unexplored, ${ }^{4}$ although tetrazines are known for their ability to react in inverse demand DielsAlder reactions, ${ }^{5}$ and for their energetic, ${ }^{6}$ dye, ${ }^{7}$ insecticide, $^{8}$ electrochemical $^{9}$ and biomedical ${ }^{10}$ properties. In particular, polyvinyltetrazines are missing in the literature, most probably because, to the best of our knowledge, vinyltetrazines are unknown. Herein, we describe the synthesis of the first derivative of this up-to-now elusive class of compounds: 3,6-divinyl-1,2,4,5-tetrazine 8. The properties of $\mathbf{8}$ and preliminary polymerization experiments are also set forth.

The first synthetic approach developed to reach compound 8 involved the elaboration of a functionalized tetrazine ring prior to further side-chain modification. An ester reduction of the readily available dimethyl $1,2,4,5-$ tetrazine-3,6-dicarboxylate ${ }^{11}$ was attempted using different reagents in order to target a tetrazine ring possessing either hydroxymethyl or formyl groups as functionalities capable of further modifications. However, lithium aluminium hydride, sodium borohydride or diisobutylaluminium hydride all led to major decomposition regardless of the conditions used. Since a ${ }^{1} \mathrm{H}$ NMR study revealed that the tetrazine ring was reduced as quickly as, or even faster than the ester groups, we assumed that the decomposition process could be the result of partial ring hydrogenation leading to a 1,2-dihydrotetrazine, followed by ring opening giving hydrophilic small entities washed away during the reaction work-up.

The second investigated pathway was based on a Stille coupling. The known 3,6-dichloro-1,2,4,5-tetrazine ${ }^{12}$ was not considered as the halogenated partner, since a recent paper $^{13}$ highlighted its unstability in palladium catalyzed conditions (Sonogashira coupling). Thus, 3,6-bis(methylsulfanyl)-1,2,4,5-tetrazine ${ }^{14}$ was used as a more stable reagent. Nevertheless, whatever the conditions, the starting tetrazine was found unreactive. As an example, a modified Stille procedure described with methylsulfanyl-substituted heterocycles ${ }^{15}$ as the substrates (tributylvinyltin, 1 eq. $\mathrm{CuBr}_{\mathrm{Me}} \mathrm{S}, 5 \mathrm{~mol} \%$ $\mathrm{Pd}\left(\mathrm{PPh}_{3}\right)_{4}$, refluxing THF or DME, 3-6 days) appeared ineffective.

This failure led us to build the tetrazine ring rather than start from a tetrazine compound. For this purpose, acrylonitrile was first tested as a precursor since several conjugated nitriles were reported to undergo cyclisation to dihydrotetrazines in the presence of hydrazine, $\mathrm{Cu}\left(\mathrm{NO}_{3}\right)_{2}$ and zinc dust. ${ }^{16}$ The cyclized product was obtained in only minor amount, standard Michael addition being the major pathway. In order to overcome this problem, a masked vinyl group was tested. Indeed, the use of appropriate phenylsulfonyl groups further treated in basic media was shown to lead to vinyl derivatives under smooth conditions. ${ }^{17}$ Thus, a cyclisation was attempted starting from nitrile $\mathbf{1}^{18}$ under the abovementioned conditions. No reaction occurred whatever the temperature used (from RT to $70{ }^{\circ} \mathrm{C}$ ) and the unreactive starting material was recovered quantitatively. This result tends to establish that the scope of this process is 
limited to a few type of precursors, such as aromatic nitriles. ${ }^{19}$ In order to increase the reactivity of 1 , its conversion into the corresponding imidate was accomplished. We focused on a Pinner methodology $y^{20}$ since the product is usually isolated in pure form following an easy work-up. Imidate hydrochloride 2 was thus obtained in $94 \%$ yield after $10 \mathrm{~h}$ at room temperature (Scheme 1).

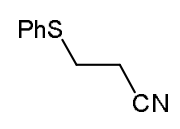

1

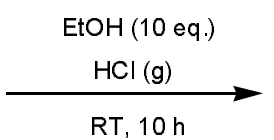

$94 \%$

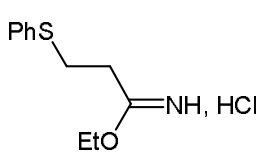

2
Scheme 1. Synthesis of imidate 2

The cyclization reaction from 2 using hydrazine hydrate proved to be the key step of the synthesis. Our first experiment using 2 equivalents of hydrazine hydrate at 0 ${ }^{\circ} \mathrm{C}$ yielded a mixture of 4-amino-1,2,4-triazole 5 and tetrazine 6 (Scheme 2 and Table 1, entry 1). This result prompted us to investigate the course of the reaction more thoroughly. Parameters such as temperature and quantity of hydrazine hydrate were varied and the products carefully isolated and characterized. ${ }^{22}$ As expected, ${ }^{23}$ higher temperatures favored the formation of compound $\mathbf{5}$. It was quantitatively obtained after simple precipitation from cyclohexane when the reaction was performed at $78{ }^{\circ} \mathrm{C}$ (Table 1 , entry 2). Using 1 equivalent of hydrazine hydrate at $20{ }^{\circ} \mathrm{C}$ (Table 1 , entry 3 ), analysis of the crude product revealed two other products: acyclic diamine $\mathbf{3}$ and 3,6-bis(2phenylsulfanylethyl)-1,2-dihydro-1,2,4,5-tetrazine 4 . We found that the latter readily oxidized to 6 during workup. Diamine 3 was by far the major product when using only 0.5 equivalent of hydrazine hydrate (Table 1 , entry 4). These results clearly evidenced that 3 is the first intermediate in the synthesis of 6 . To the best of our knowledge, this kind of acyclic intermediate has never been isolated in hydrazine mediated syntheses of tetrazines from imidates or related compounds.

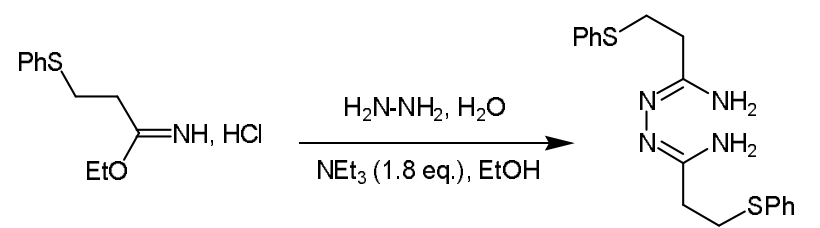

2

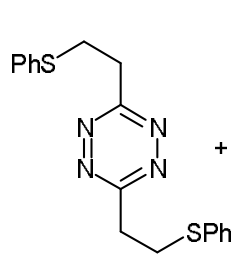

6

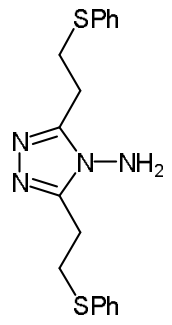

5
3

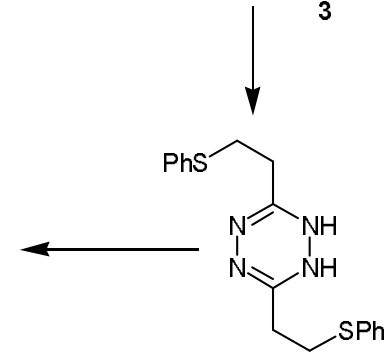

4
Scheme 2. Cyclization reaction of imidate 2 Table 1. Cyclization reaction of imidate 2 using hydrazine hydrate

\begin{tabular}{llllllll} 
Entry & $\begin{array}{l}\mathrm{N}_{2} \mathrm{H}_{4} \\
(\mathrm{eq})\end{array}$ & $\begin{array}{l}\mathrm{T} \\
\left({ }^{\circ} \mathrm{C}\right)\end{array}$ & $\begin{array}{l}\mathrm{t} \\
(\mathrm{h})\end{array}$ & $\mathbf{3}$ & $\mathbf{4}$ & $\mathbf{5}$ & $\mathbf{6}$ \\
\hline 1 & 2 & 0 & 4 & 0 & 0 & $24^{a}$ & $30^{a}$ \\
2 & 2 & 78 & 19 & 0 & 0 & $100^{a}$ & 0 \\
3 & 1 & 20 & 6 & 43 & 26 & 19 & 12 \\
$4^{b}$ & 0.5 & 20 & 4 & 78 & 0 & 0 & 7 \\
$a$ & Isolated product. \\
$b$ & : $15 \%$ of starting material were detected in the crude \\
mixture.
\end{tabular}

According to standard procedures ${ }^{24}$ oxidation of 6 was then performed using $4.1 \mathrm{eq}$. of $m$-CPBA at low temperature, leading to disulfone 7 (Scheme 3). ${ }^{25}$ As expected, this occurred chemoselectively since no nitrogen oxidation was observed after treatment. The subsequent 1,2-elimination of the phenylsulfonyl end-chain was expected to generate vinyl groups as reported earlier. Among the large palette of bases usually employed, ${ }^{17}$ we focused on the use of $t$-BuOK in THF for practical reasons. Even a slight excess of potassium tertbutoxide resulted in low yields. We therefore settled for an amount of base of 1.9 equivalent, thus ensuring a reasonable conversion rate (the monovinyl derivative was also evidenced) without formation of further byproducts. After purification, the target compound 3,6divinyl-1,2,4,5-tetrazine 8 was obtained in a $42 \%$ yield as a volatile, pink oil which solidified in the freezer (Scheme 3). ${ }^{26}$

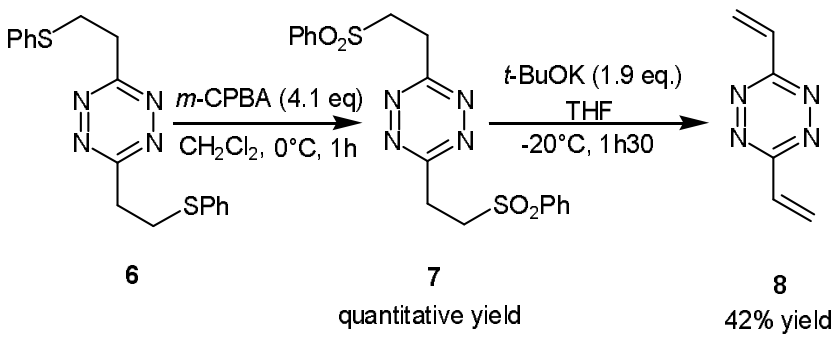

Scheme 3. Oxidation of tetrazine 6 to vinyltetrazine 8

Polymerization experiments were then carried out, using radical or anionic initiation, but without success. The failure of homopolymerization and even copolymerization reactions suggested that $\mathbf{8}$ could act as a radical inhibitor. However, EPR experiments did not confirm this assumption. Therefore, the stability of $\mathbf{8}$ in various conditions was checked. When 3,6-divinyl-1,2,4,5tetrazine was reacted under UV irradiation at $-10{ }^{\circ} \mathrm{C}$ with $11 \mathrm{~mol} \%$. of Irgacure 651 as the radical initiator, a total decomposition of the starting material occurred as indicated by ${ }^{1} \mathrm{H}$ NMR. Since a further experiment performed under similar conditions without any radical initiator led to entire recovery of $\mathbf{8}$, its radical sensitivity was evidenced. The second investigation focused on the stability of 8 with increasing temperature. 3,6-Divinyl1,2,4,5-tetrazine was left unchanged after heating during 5 days at $40{ }^{\circ} \mathrm{C}\left(0.1 \mathrm{M}\right.$ in $\left.\mathrm{CDCl}_{3}\right)$, as evidenced by ${ }^{1} \mathrm{H}$ NMR. On the contrary, when the temperature was in- 
creased $\left(80^{\circ} \mathrm{C}\right.$ in DMF) an insoluble material started to appear. The same solid was recovered when 8 was allowed to stand without caution at room temperature. Since the brownish solid was found insoluble in every solvents, its characterization remained cumbersome. We anticipated that this could be the homopolymer of $\mathbf{8}$, but elemental analyses revealed that the nitrogen content of the solid was too low $(28.1 \%$ instead of the expected $41.8 \%$ ). These results could be indicative of [4+2] cycloadditions with loss of $\mathrm{N}_{2}$, a well-known process in the tetrazine series, ${ }^{5}$ leading to a cross-linked polypyridazine network.

In summary, we have described the synthesis of 3,6divinyl-1,2,4,5-tetrazine, the first reported example to date of a vinyltetrazine. Compound 8 can be seen as a tetraaza analogue of the widely used 1,4-divinylbenzene. Therefore, although our preliminary studies showed a limited stability at high temperatures and in the presence of radicals, 8 is believed to be a useful building block in the synthesis of new tetrazine-based molecules and materials. Moreover, 8 stands as an interesting substrate for Diels-Alder reactions, since both the tetrazine ring and the lateral double bonds could participate in such processes.

\section{Acknowledgment}

Authors wish to thank Dr Pierre Guenot (CRMPO, Université de Rennes 1) for his assistance in the 3,6-divinyl-1,2,4,5-tetrazine stability investigations

\section{References}

(1) Kizhnyaev, V. N.; Vereshchagin, L. I. Russ. Chem. Rev. 2003, 72, 143-164 and references cited therein.

(2) (a) Kizhnyaev, V. N.; Pokatilov, F. A.; Tsypina, N. A.; Ratovskii, G. V:; Vereshchagin, L. I.; Smirnov, A. I. Russ. J. Org. Chem. 2002, 38, 1056-1059. (b) Wouters, G.; Smets, G. Makromol. Chem. 1982, 183, 1861-1868. (c) Attaryan, O. S.; Asratyan, G. V: Eliazyan, G. A.; Darbinyan, E. G.; Matsoyan, S. G. Arm. Khim. Zh. 1986, 39, 630-635. (d) Toppet, S.; Wouters, G.; Smets, G. J. Polym. Sci. Polym. Lett. 1976, 14, 389-394.

(3) (a) American Cyanamid Company, GB Patent GB1963-935787. (b) Schaefer, F. C.; Peters, G A.; US Patent US 1958-2845422. (c) Yuki, Y.; Kakurai, T.; Nogushi, T.; Hiramatsu, N. Kobbunshi Kugaku 1969, 26, 134-140 (d) Yuki, Y:; Kakurai, T:; Nogushi, T. Kôbunshi Kugaku 1969, 26, 141-147. (e) Overberger, C. G.; Shapiro, S. L. $J$. Am. Chem. Soc. 1954, 76, 1061-1065. (f) Fukui, K.; Kitano, H.; Mizuta, M.; Osaka, T.; Kanai, K. Japanese Patent JP 1970-44025793.

(4) (a) Audebert, P.; Sadki, S.; Miomandre, F.; Clavier, G. Electrochem. Commun. 2004, 6, 144-147. (b) Topp, K. D.; Grote, M. React. Funct. Polym. 1996, 31, 117-136. (c) Zhang, B. W.; Fischer, K.; Bieniek, D.; Kettrup, A. React. Funct. Polym. 1994, 24, 49-58. (d) Stoicescu-Crivetz, L.; Mantaluta, E.; Neamtu, G.; Zugravescu, I. J. Pol. Sci.: Part C. 1969, 22, 761-771. (e) Stoicescu-Crivetz, L.; Mantaluta, E.; Neamtu, G; Zugravescu, I. Rev. Roum. Chim. 1966, 11, 1127-1134. (f) Konishi, K.; Kobota, J.; Kotone, A.; Nakane, Y. Japanese Patent JP 1972-7234499.

(5) (a) Hamasaki, A.; Ducray, R.; Boger, D. L. J. Org. Chem. 2006, 71, 185-193. (b) Soenen, D. R.; Zimpleman, J. M.; Boger, D. L. J. Org. Chem. 2003, 68, 3593-3598. (c) Wan,
Z. K.: Woo, G. H. C.: Snyder, J. K. Tetrahedron 2001, 57, 5497-5507. (d) Kotschy, A.; Novák, Z.; Vincze, Z.; Smith, D. M: Hajós, G. Tetrahedron Lett. 1999, 40, 6313-6316. (e) Seitz, G.; Overheu, W. Arch. Pharm. 1977, 310, 936938.

(6) (a) Chavez, D. E.; Hiskey, M. A.; Gilardi, R. D. Org. Lett. 2004, 6, 2889-2891. (b) Talawar, M. B.; Sivabalan, R.; Senthilkumar, N.; Prabhu, G.; Asthana, S. N. J. Hazard. Mater. 2004, 113, 11-25. (c) Pagoria, P. F.; Lee, G. S.; Mitchell, A. R.; Schmidt, R. D. Thermochimica Acta 2002, 384, 187-204. (d) Oxley, J. C.; Smith, J. L.; Chen, H. Thermochimica Acta 2002, 384, 91-99. (e) Chavez, D. E.; Hiskey, M. A.; Gilardi, R. D. Angew. Chem., Int. Ed. 2000, 39, 1791-1793.

(7) Kotone A.; Hoda M.; (Sakai Chem. Int. Co. Ltd) Japanese Patent JP 1971-24002.

(8) Tsuda S.; Manabe Y.; Tsuji K.; (Sumitomo Chem. Co. Ltd) Japanese Patent JP 1988-107254.

(9) Audebert, P.; Sadki, S.; Miomandre, F.; Clavier, G.; Vernières, M.-C.: Saoud, M.; Hapiot, P. New. J. Chem 2004, 28, 387-392.

(10) (a) Rao, G. W.; Hu, W. X. Bioorg. Med. Chem. Lett. 2005, 15, 3174-3176. (b) Hu, W. X.; Rao, G W:; Sun Y. Q. Bioorg. Med. Chem. Lett. 2004, 14, 1177-1181.

(11) Boger, D. L.; Coleman, R. S.; Panek, J. S. J. Org. Chem. 1985, 50, 5377-5379.

(12) (a) Schirmer, U.; Wuerzer, B.; Meyer, N.; Neugebauer, F. A.; Fischer, H. Ger. Offen. DE 1987-350821411. (b) Chavez, D. E.; Hiskey, M. A. J. Energetic Mater. 1999, 17 , 357-377. (c) Novak, Z.; Bostai, B.; Csekei, M.; Lorincz, K: Kotschy, A. Heterocycles 2003, 60, 12, 2653-2668.

(13) Novak, Z.; Kotschy, A. Org. Lett. 2003, 19, 3495-3497.

(14) Sandstrom, J. Acta Chem. Scand. 1961, 15, 1575-1582.

(15) Alphonse, F. A.; Suzenet, F.; Keromnes, A.; Lebret, B.; Guillaumet, G. Org. Lett. 2003, 5, 803-805.

(16) Lim, C. L.; Pyo, S. H.; Kim, T. Y.; Yim, E. S.; Han, B. H. Bull. Korean Chem. Soc. 1995, 16, 374-377.

(17) (a) Simpkins, N. S. In Sulfones in organic synthesis, Tetrahedron Org. Chem. Series, Ed. Baldwin, J. E., Frs. and Pd. Magnus, Pergamon Press, Oxford, 1993, Vol. 10. (b) Julia, M.; Arnould, D. Bull. Soc. Chim. Fr. 1973, 2, 746-750. (c) Colter, A. K.; Miller Jr, R. E. J. Org. Chem. 1971, 36 , 1898-1903. (d) Hwang, S. H.; Kurth, M. J. Tetrahedron Lett. 2002, 43, 53-56. (e) Giovannini, R.; Marcantoni, E.; Pietrini, M. Tetrahedron Lett. 1998, 39, 5827-5830. (f) Baker-Glenn, C. A. G.; Barrett, A. G. M.; Gray, A. A.; Procopiou, P. A.; Ruston, M. Tetrahedron Lett. 2005, 46, 7427-7430.

(18) Renard, M.; Ghosez, L. A. Tetrahedron 2001, 57, 2597 2608.

(19) Soloducho, J.; Doskocz, J.; Cabaj, J.; Roszak, S. Tetrahedron 2003, 59, 4761-4766.

(20) (a) Weintraub, L.; Oles, S. R.; Kalish, N. J.Org. Chem. 1968, 33, 1679-1681. (b) Butler, R. C. M.; Chapleo, C. B.; Myers, P. L.; Welburn, A. P. J. Heterocycl. Chem. 1985, 22, 177-181.

(21) Ethyle 3-phenylsulfanylpropionimido ester hydrochloride (2). A steady stream of gaseous hydrogen chloride was bubbled at room temperature for $10 \mathrm{~h}$ through a stirred ethanolic solution ( $7.3 \mathrm{~mL}, 0.125 \mathrm{~mol})$ of propionitrile 1 $(2.02 \mathrm{~g}, 12.3 \mathrm{mmol})$. A white powder gradually precipitated. The solvent was removed under vacuum and the crude precipitate was triturated three times with anhydrous ether and finally filtered leading to compound 2 as a white powder ( $94 \%$ yield). $\mathrm{mp} 74-75^{\circ} \mathrm{C} ;{ }^{1} \mathrm{H}$ NMR $\left(\mathrm{CDCl}_{3}\right) \delta$ $12.50(\mathrm{~s}, 1 \mathrm{H}), 11.60(\mathrm{~s}, 1 \mathrm{H}), 7.41-7.27(\mathrm{~m}, 5 \mathrm{H}), 4.56(\mathrm{q}, J=$ $6.9 \mathrm{~Hz}, 2 \mathrm{H}), 3.30(\mathrm{t}, J=6.6 \mathrm{~Hz}, 2 \mathrm{H}), 3.06(\mathrm{t}, J=6.6 \mathrm{~Hz}$, $2 \mathrm{H}), 1.44(\mathrm{t}, J=6.9 \mathrm{~Hz}, 3 \mathrm{H}) ;{ }^{13} \mathrm{C} \mathrm{NMR}\left(\mathrm{CDCl}_{3}\right) \delta 177.0$, $133.7,130.9,129.1,126.4,70.9,33.4,28.8,13.4 ; \mathrm{IR}(\mathrm{KBr})$ 
$v_{\max } 3078,2951,2234,1692,1384,975 \mathrm{~cm}^{-1}$; LC/MS $\left(\mathrm{AP}+, \mathrm{CH}_{3} \mathrm{CN} / \mathrm{H}_{2} \mathrm{O} 80 / 20\right): 2.67 \mathrm{~min}, 246.7(\mathrm{M}+1)$ and 210.3 (M-Cl); HRMS (FI) calcd for $\mathrm{C}_{11} \mathrm{H}_{15} \mathrm{ONS}$ (M-HCl) 209.0874, found 209.0875.

(22) General cyclization procedure. Imidoester hydrochloride $2(6.17 \mathrm{~g}, 25.1 \mathrm{mmol})$ was dissolved in ethanol $(0.16 \mathrm{M})$ under a nitrogen atmosphere and the solution was brought to $-10^{\circ} \mathrm{C}$. Then triethylamine and hydrazine monohydrate $(2.5 \mathrm{~mL}, 51.4 \mathrm{mmol}$ ) were successively added to the solution and the mixture was allowed to stir (conditions: see table 1). The mixture was concentrated under vacuum and the slightly pink crude solid obtained was further dissolved in ethyl acetate and washed with water. The aqueous layer was then extracted with AcOEt and the resulting organic layer was washed with brine, dried over $\mathrm{MgSO}_{4}$, filtered and concentrated under vacuum. Depending on reactional conditions the products could be obtained. Compound $\mathbf{5}$ was isolated after simple precipitation from cyclohexane, whereas 3,4 , and $\mathbf{6}$ were obtained after chromatography over silica gel (cyclohexane/AcOEt 7/1). N'-[1-amino-3(phenylsulfanyl)propylid-1-ene]-3-

(phenylthio)propanehydrazonamide (3). $\mathrm{mp} 75^{\circ} \mathrm{C}$ (white crystals); ${ }^{1} \mathrm{H} \mathrm{NMR}\left(\mathrm{CDCl}_{3}\right) \delta 7.88-7.15(\mathrm{~m}, 10 \mathrm{H})$, 5.43 and 5.09 (br s, $4 \mathrm{H}), 3.24(\mathrm{t}, J=7.4,4 \mathrm{H}), 2.55(\mathrm{t}, J=$ $7.4,4 \mathrm{H}) ;{ }^{13} \mathrm{C} \mathrm{NMR}\left(\mathrm{CDCl}_{3}\right) \delta 155.8,135.5,129.5,129.0$, $126.3,32.9,30.8 ; \mathrm{R}(\mathrm{KBr}) v_{\max } 3450,3337,3060,2960$, $1633,1583,1482,1444,1356,1287,910 \mathrm{~cm}^{-1}$. LC/MS $\left(\mathrm{AP}+, \mathrm{CH}_{3} \mathrm{CN} / \mathrm{H}_{2} \mathrm{O}\right.$ 80/20): 3.40, min, 358.9. 3,6-Bis-(2phenylsulfanylethyl)-1,2-dihydro-1,2,4,5-tetrazine (4). $\mathrm{mp} 151{ }^{\circ} \mathrm{C}$ (white crystals); ${ }^{1} \mathrm{H}$ NMR (DMSO- $\left.d_{6}\right) \delta 7.96$ (s, $2 \mathrm{H}), 7.37-7.26(\mathrm{~m}, 8 \mathrm{H}), 7.24-7.12(\mathrm{~m}, 2 \mathrm{H}), 3.10(\mathrm{t}, J=7.6$, $4 \mathrm{H}), 2.30(\mathrm{t}, J=7.6,4 \mathrm{H}) ;{ }^{13} \mathrm{C} \mathrm{NMR}$ (DMSO- $\left.d_{6}\right) \delta 148.5$, 135.7, 129.1, 128.1, 125.7, 29.8, 28.2; IR $v_{\max } 3243,3054$, $1677,1575,1476,1436,1400,1287,1257,1242,1208$, $1165,1086,1020 \mathrm{~cm}^{-1}$; LC/MS (AP+, $\mathrm{CH}_{3} \mathrm{CN} / \mathrm{H}_{2} \mathrm{O} 50 / 50$ ): $7.15 \mathrm{~min}, 356.9$. 4-Amino-3,5-bis(2-phenylsulfanylethyl)1,2,4-triazole (5). $\mathrm{mp} 80-82{ }^{\circ} \mathrm{C}$ (white crystals); ${ }^{1} \mathrm{H} N \mathrm{NR}$ $\left(\right.$ DMSO-d $\left._{6}\right) \delta 7.40-7.16(\mathrm{~m}, 10 \mathrm{H}), 5.63(\mathrm{~s}, 2 \mathrm{H}), 3.31(\mathrm{t}, J=$ $7.0 \mathrm{~Hz}, 4 \mathrm{H}), 2.98(\mathrm{t}, J=7.0 \mathrm{~Hz}, 4 \mathrm{H}) ;{ }^{13} \mathrm{C} \mathrm{NMR}\left(\mathrm{CDCl}_{3}\right) \delta$ $153.5,134.9,129.0,128.9,126.3,31.4,24.6 ; \mathrm{R}(\mathrm{KBr}) v_{\max }$ 3444, 3042, 2991, 1633, 1583, 1482, 1438, 1231, 1205, $790 \mathrm{~cm}^{-1}$; LC/MS (AP+, $\left.\mathrm{CH}_{3} \mathrm{CN} / \mathrm{H}_{2} \mathrm{O} 80 / 20\right): 2.49 \mathrm{~min}$, $356.6\left(\mathrm{MH}^{\dagger}\right)$; Anal calcd for $\mathrm{C}_{18} \mathrm{H}_{20} \mathrm{~N}_{4} \mathrm{~S}_{2}$ : C 60.64, H 5.65, N 15.72, S 17.93, found: C 60.31, H 5.81, N 15.67, S 17.71. 3,6-Bis(2-phenylsulfanylethyl)-1,2,4,5-tetrazine (6). $\mathrm{mp} 55^{\circ} \mathrm{C}$ (pink crystals); ${ }^{1} \mathrm{H} \mathrm{NMR}\left(\mathrm{CDCl}_{3}\right) \delta 7.42-$ $7.35(\mathrm{~m}, 4 \mathrm{H}), 7.32-7.25(\mathrm{~m}, 4 \mathrm{H}), 7.24-7.17(\mathrm{~m}, 2 \mathrm{H}), 3.60$ $(\mathrm{t}, J=6.8 \mathrm{~Hz}, 4 \mathrm{H}), 3.52(\mathrm{t}, J=6.8 \mathrm{~Hz}, 4 \mathrm{H}) ;{ }^{13} \mathrm{C} \mathrm{NMR}$ $\left(\mathrm{CDCl}_{3}\right) \delta 168.4,134.5,130.5,129.0,126.7,34.7,31.8 ; \mathrm{IR}$ $(\mathrm{KBr}) v_{\max } 3023,2406,1520,1425,1356,1218,1048,929$ $\mathrm{cm}^{-1}$; LC/MS (AP+, $\mathrm{CH}_{3} \mathrm{CN} / \mathrm{H}_{2} \mathrm{O}$ 80/20): $3.12 \mathrm{~min}, 355.2$ $\left(\mathrm{MH}^{+}\right)$; HRMS (Cl) calcd for $\mathrm{C}_{18} \mathrm{H}_{19} \mathrm{~N}_{4} \mathrm{~S}_{2}\left(\mathrm{MH}^{+}\right)$355.1051, found 355.1043

(23) Katritzky, A. R.; Rees, C. W.; Scriven, E. F. V. Comprehensive Heterocyclic Chemistry II; Pergamon press: Oxford, 1996, Vol. 6, 949

(24) Panek, J. S.; Zhu, B. Tetrahedron Lett. 1996, 37, 81518154 .

(25) 3,6-Bis(2-phenylsulfonylethyl)-1,2,4,5-tetrazine (7). Tetrazine $6(1.86 \mathrm{~g}, 5.25 \mathrm{mmol})$ was dissolved in $\mathrm{CH}_{2} \mathrm{Cl}_{2}$ $(0.05 \mathrm{M})$ and the solution was cooled to $-10{ }^{\circ} \mathrm{C}$. A solution of $m$-CPBA $(5.38 \mathrm{~g}, 21.8 \mathrm{mmol})$ in $\mathrm{CH}_{2} \mathrm{Cl}_{2}(0.25 \mathrm{M})$ was added over a period of $1 \mathrm{~h}$ (the temperature was kept below $0{ }^{\circ} \mathrm{C}$ ), after which TLC analysis indicated completion of the reaction (cyclohexane/AcOEt $7 / 1, \mathrm{R}_{\mathrm{f}}(\boldsymbol{6})=0.37$, pink spot; $R_{f}(7)=0$, pink spot). The mixture was successively washed with a solution of $\mathrm{NaHSO}_{3}(10 \%)$ and a solution of $\mathrm{NaHCO}_{3}(10 \%)$. The organic layer was then washed with saturated $\mathrm{NaCl}$ solution, dried over $\mathrm{MgSO}_{4}$ and filtered.
Product 7 was finally obtained as a pink powder $(2.19 \mathrm{~g}$ $100 \%$ ) after concentration under vacuum. $\mathrm{mp} 183-185^{\circ} \mathrm{C}$; ${ }^{1} \mathrm{H}$ NMR $\left(\mathrm{DMSO}-d_{6}\right) \delta$ 7.92-7.88 $(\mathrm{m}, 4 \mathrm{H}), 7.74-7.60(\mathrm{~m}$, 2H), 7.71-7.62 (m, 4H), $3.93(\mathrm{t}, J=7.6 \mathrm{~Hz}, 4 \mathrm{H}), 3.44(\mathrm{t}, J$ $=7.6 \mathrm{~Hz}, 4 \mathrm{H}) ;{ }^{13} \mathrm{C} \mathrm{NMR}\left(\mathrm{DMSO}-d_{6}\right) \delta 167.0,138.2,134.1$, $129.6,127.9,51.9,28.1$. IR (KBr) $v_{\max } 1448,1427,1395$, 1310, 1294, 1269, 1148, 1083, 1047, 1033, 996, 980, 906, $778,745,711 \mathrm{~cm}^{-1}$; LC/MS (AP+, $\mathrm{CH}_{3} \mathrm{CN} / \mathrm{H}_{2} \mathrm{O} 50 / 50$ ): $5.72 \mathrm{~min}, 418.9\left(\mathrm{MH}^{+}\right)$; Anal. calcd for $\mathrm{C}_{18} \mathrm{H}_{18} \mathrm{~N}_{4} \mathrm{~S}_{2} \mathrm{O}_{4}: \mathrm{C}$ 51.66, H 4.34, N 13.39, found: C 51.98, H 4.40, N 13.55; HRMS (CI) calcd for $\mathrm{C}_{18} \mathrm{H}_{19} \mathrm{~N}_{4} \mathrm{~S}_{2} \mathrm{O}_{4}\left(\mathrm{MH}^{+}\right) 419.0848$, found 419.0854

(26) 3,6-Divinyl-1,2,4,5-tetrazine (8). To a stirred solution of $s$ tetrazine $7(600 \mathrm{mg}, 1.43 \mathrm{mmol})$ in dry THF $(120 \mathrm{~mL})$ was added $t$-BuOK ( $307 \mathrm{mg}, 2.73 \mathrm{mmol}$ ) at $-20^{\circ} \mathrm{C}$ for $1.5 \mathrm{~h}$. The reaction was followed by TLC analysis (cyclohexane/AcOEt $4 / 6, \mathrm{R}_{\mathrm{f}}(\mathbf{7})=0.45, \mathrm{R}_{\mathrm{f}}(\mathbf{8})=0.95$ ). The solution was finally diluted with $150 \mathrm{~mL}$ of cooled $\mathrm{Et}_{2} \mathrm{O}$, filtered and washed with a solution of $\mathrm{NaHCO}_{3}(10 \%)(2 \times 100$ $\mathrm{mL})$. The organic layer was then washed with brine $(150$ $\mathrm{mL}$ ), dried over $\mathrm{MgSO}_{4}$ and further concentrated under a controlled vacuum because of its volatility. Product 8 was purified over a silica gel column (pentane/Et $\mathrm{t}_{2} \mathrm{O} 80 / 20, \mathrm{R}_{\mathrm{f}}=$ $0.96)$, obtained as a dark pink oil ( $81 \mathrm{mg}, 42 \%$ yield $)$ and kept in the freezer. ${ }^{1} \mathrm{H} \mathrm{NMR}\left(\mathrm{CD}_{2} \mathrm{Cl}_{2}\right) \delta 7.17(\mathrm{dd}, J=10.8$ $\mathrm{Hz}, J=17.6 \mathrm{~Hz}, 2 \mathrm{H}), 7.01(\mathrm{dd}, J=1.2 \mathrm{~Hz}, J=17.6 \mathrm{~Hz}$, $2 \mathrm{H}), 6.02(\mathrm{dd}, J=1.2 \mathrm{~Hz}, J=10.8 \mathrm{~Hz}, 2 \mathrm{H}) ;{ }^{13} \mathrm{C} \mathrm{NMR}$ $\left(\mathrm{CD}_{2} \mathrm{Cl}_{2}\right) \delta 164.2,131.0,127.8$; $\mathrm{R}(\mathrm{KBr}) \mathrm{v}_{\max } 1632,1434$, $1364,1343,1259,1075,1032,984,950,902,711 \mathrm{~cm}^{-1}$. LC/MS (AP+, MeOH): $0.04 \mathrm{~min}, 135.3\left(\mathrm{MH}^{+}\right)$; HRMS (FI) calcd for $\mathrm{C}_{6} \mathrm{H}_{6} \mathrm{~N}_{4}\left(\mathrm{M}^{+}\right) 134.0592$, found 134.0590 . 
Please place the graphical abstract and short title of the article here. The short title will be used as a running header.

Synthesis of 3,6-Divinyl-1,2,4,5-Tetrazine
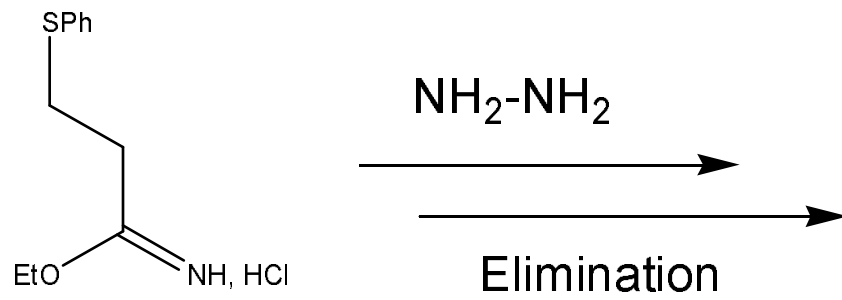

Elimination

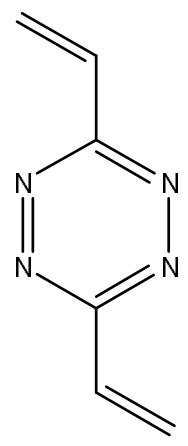

2

8 